\section{Copy number variation of the REX01L1 gene cluster; euchromatic deletion variant or susceptibility factor?}

European Journal of Human Genetics (2017) 25, 8-9; doi:10.1038/ejhg.2016.104; published online 3 August 2016

Relatively little is known about the function of the RNA exonuclease 1 homologue (Saccharomyces cerevisiae)-like 1 gene (REXO1L1) in humans and only two titles in PubMed contain the REXO1L1 acronym. Evidence published in this journal indicated that copy number variation $(\mathrm{CNV})$ of the REXO1L1 gene and pseudogene cluster was common and likely to be benign. ${ }^{1}$ In contrast, it has been proposed that de novo heterozygous deletion of the REXO1L1 cluster in a single patient is responsible for a new microdeletion syndrome including dysmorphic features, cleft palate, incomplete spina bifida, dyspraxia, global developmental delay, growth retardation and gastrointestinal malabsorption. ${ }^{2}$ Here we argue that determining the possible clinical significance of large-scale CNV requires the application of accurate quantitative techniques to both individual families and large cohorts of affected and unaffected individuals.

\section{MICRODELETION OR EUCHROMATIC DELETION VARIANT?}

The REXO1L1 gene and pseudogene cluster lies within a $12 \mathrm{~kb}$ tandem repeat in band $8 \mathrm{q} 21.2$ that is one of the largest variable number tandem repeat arrays in the human genome. ${ }^{3}$ D'Apice et al. ${ }^{2}$ used loss of hybridisation signal from a single bacterial artificial chromosome (BAC) to imply that the whole REXO1L1 cluster had been deleted but their BAC (RP11-96G1) only partially overlaps this region (Supplementary Figure 1) and residual copies of the $12 \mathrm{~kb}$ repeat might not have been detected using FISH. BAC RP11-96G1 is also one of the commonest benign $\mathrm{CNV}$ s found using $\mathrm{BAC}$ aCGH ${ }^{4}$ and subject to loss, gain and inversion in the database of genomic variants.

The REXO1L1 gene has no haploinsufficiency likelihood score (Dataset 2) ${ }^{5}$ and, even if the REXO1L1 cluster was deleted, the median diploid copy number of the REXO1L1 gene was 166 and ranged from 97 to 277 copies in 216 controls $^{1}$ and 165 HapMap individuals ${ }^{6}$ using digital NanoString technology (Supplementary Figure 2). If the patient studied by D'Apice et al. ${ }^{2}$ had half the lowest observed diploid copy number of 97, the remaining copies on the homologous chromosome 8 would be expected to compensate for the loss of the cluster from the chromosome 8 carrying the deletion. Using the less accurate method of real-time quantitative $\mathrm{PCR}^{7}$ with calibrator plasmids as controls, D'Apice et al. ${ }^{2}$ reported diploid copy numbers that were in an order of magnitude lower with 16-24 diploid copies in 60 controls and 8 in their deleted patient.
High copy number of the REXO1L1 cluster can result in a common cytogenetically visible euchromatic variant (EV) that was not thought to be linked to the phenotypes in seven patients with reproductive problems (4), autism (1), a history of intellectual difficulties and cystic fibrosis (1) or congenital anomalies better explained by mosaic trisomy $9(1) .{ }^{1}$ In all seven reported 8q21.2 EV carriers, FISH signals from the variant chromosomes were approximately twice as large as those on the homologous chromosomes. ${ }^{1}$ These simple $\sim 2$ :1 ratios suggest that the extreme high and low ends of the otherwise continuous REXO1L1 copy number distribution might be due to large reciprocal duplications and deletions of the REXO1L1 cluster that are cytogenetically visible as EVs (Supplementary Figure 2). As gains of RP11-96G1 were far more frequent than losses in the healthy populations analysed by Redon et al, ${ }^{8}$ D'Apice et al. ${ }^{2}$ may have found a rare benign euchromatic deletion variant of $8 \mathrm{q} 21.2$ rather than a novel microdeletion. If not due to dosage, any possible effects of a deletion would require alternative mechanisms such as enhancer adoption by genes flanking the REXO1L1 cluster $^{7}$ or the release of other genes from epigenetic control. ${ }^{6}$

\section{SUSCEPTIBILITY FACTORS}

There is increasing evidence for a role for recurrent CNVs in infectious disease, including lower copy number of the chemokine CC motif ligand 3-like 1 gene with susceptibility to chronic HCV infection. $^{7}$ As immunological reaction against the human REXO1L1 gene product is a marker of HCV infection, a role for REXO1L1 copy number in HCV infection was suggested. ${ }^{1}$ D'Apice et al. ${ }^{2}$ found increased genetic instability and apoptosis in their patient fibroblasts after treatment with DNA-damaging agents and, as REXO1L1 is a 3'-5' exonuclease member of the DEDD superfamily which might control HBV and RNA virus infections, speculated that low REXO1L1 copy number might predispose and high numbers protect against viral infection.

The patient of D'Apice et al. ${ }^{2}$ also had malabsorption syndrome with inflammatory infiltrates of the gastrointestinal tract and persistent diarrhoea for which a gluten-free diet provided temporary resolution. As other large-scale CNVs have been associated with inflammatory diseases $^{7,9}$ it is conceivable that low REXO1L1 copy number might predispose to inflammatory gastrointestinal conditions that include celiac, Crohn's and irritable bowel syndrome. The higher frequency of RP11-96G1 gains than losses ${ }^{8}$ and the 0.7 skew against lower copy numbers among controls ${ }^{1}$ suggest that there may be factors that select against low REXO1L1 cluster copy number.

\section{CONCLUSION}

On the current evidence, it is doubtful that all the phenotypes observed by D'Apice et al. ${ }^{2}$ are due to deletion of the REXO1L1 gene and pseudogene cluster and more likely that these authors have found a benign EV representing a low extreme of normal CNV. Understanding the possible role of this variation in gastrointestinal impairment and viral infection would be aided by more extensive testing of copy number in affected cohorts and controls with accurate quantitative techniques such as digital copy number estimation ${ }^{1,6}$ or massively parallel sequencing. ${ }^{10}$ Phase and haploid copy number could also be established in multigenerational families including those 
segregating outliers that could still be detected cytogenetically as EVs in routine clinical laboratories.

\section{WEB RESOURCES}

DGV (Database of Genome Variants): http://dgv.tcag.ca/dgv/app/home UCSC web browser: http://genome.ucsc.edu/

\section{CONFLICT OF INTEREST}

The authors declare no conflict of interest.

John CK Barber ${ }^{\star 1}$, Andrew J Sharp ${ }^{2}$, Edward J Hollox ${ }^{3}$ and Christine Tyson ${ }^{4}$

${ }^{1}$ Department of Human Genetics and Genomic Medicine, Faculty of Medicine, University of Southampton, Southampton General Hospital, Southampton, UK;

${ }^{2}$ Department of Genetics and Genomic Sciences, Mount Sinai School of Medicine, New York City, NY, USA;

${ }^{3}$ Department of Genetics, University of Leicester, Leicester, UK; ${ }^{4}$ Cytogenetics Laboratory, Department of Pathology, Royal Columbian Hospital, New Westminster, British Columbia, Canada E-mail: john.barber@soton.ac.uk
1 Tyson C, Sharp AJ, Hrynchak M et al: Expansion of a 12- kb VNTR containing the REXO1L1 gene cluster underlies the microscopically visible euchromatic variant of 8q21.2. Eur J Hum Genet 2014; 22: 458-463.

2 D'Apice MR, Novelli A, di Masi A et al: Deletion of REXO1L1 locus in a patient with malabsorption syndrome, growth retardation, and dysmorphic features: a novel recognizable microdeletion syndrome? BMC Med Genet 2015; 16: 20.

3 Warburton PE, Hasson D, Guillem F et al: Analysis of the largest tandemly repeated DNA families in the human genome. BMC Genomics 2008; 9: 533.

4 Whitby $\mathrm{H}$, Tsalenko A, Aston $\mathrm{E}$ et al: Benign copy number changes in clinical cytogenetic diagnostics by array CGH. Cytogenet Genome Res 2008; 123: 94-101.

5 Huang N, Lee I, Marcotte EM, Hurles ME: Characterising and predicting haploinsufficiency in the human genome. PLoS Genet 2010; 6: e1001154.

6 Brahmachary M, Guilmatre A, Quilez J et al: Digital genotyping of macrosatellites and multicopy genes reveals novel biological functions associated with copy number variation of large tandem repeats. PLoS Genet 2014; 6: e1004418.

7 Hollox EJ, Hoh BP: Human gene copy number variation and infectious disease. Hum Genet 2014; 133: 1217-1233.

8 Redon R, Ishikawa S, Fitch KR et al: Global variation in copy number in the human genome. Nature 2006; 444: 444-454.

9 Fernandez-Jimenez N, Castellanos-Rubio A, Plaza-Izurieta $L$ et al: Analysis of betadefensin and Toll-like receptor gene copy number variation in celiac disease. Hum Immunol 2010; 71: 833-836.

10 Nuttle X, Itsara A, Shendure J, Eichler EE: Resolving genomic disorder-associated breakpoints within segmental DNA duplications using massively parallel sequencing. Nat Protoc 2014; 9: 1496-1513.

Supplementary Information accompanies this paper on European Journal of Human Genetics website (http://www.nature.com/ejhg) 